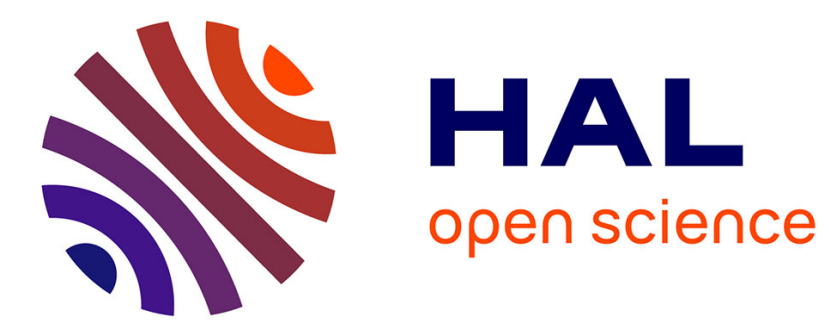

\title{
Extreme anomalous centrifugal distortion in methylene
} Laurent H Coudert

\section{To cite this version:}

Laurent H Coudert. Extreme anomalous centrifugal distortion in methylene. Journal of Chemical Physics, 2020, 153 (14), pp.144115. 10.1063/5.0026162 . hal-02966780

\section{HAL Id: hal-02966780 \\ https://hal.science/hal-02966780}

Submitted on 14 Oct 2020

HAL is a multi-disciplinary open access archive for the deposit and dissemination of scientific research documents, whether they are published or not. The documents may come from teaching and research institutions in France or abroad, or from public or private research centers.
L'archive ouverte pluridisciplinaire HAL, est destinée au dépôt et à la diffusion de documents scientifiques de niveau recherche, publiés ou non, émanant des établissements d'enseignement et de recherche français ou étrangers, des laboratoires publics ou privés. 


\title{
Extreme anomalous centrifugal distortion in methylene
}

\author{
Laurent H. Coudert* \\ Université Paris-Saclay, CNRS, Institut des Sciences Moléculaires d'Orsay, 91405, Orsay, France
}

(Dated: October 14, 2020)

\begin{abstract}
A new treatment is presented to account for the extreme anomalous centrifugal distortion displayed by the open-shell methylene radical. This new treatment is based on a four-dimensional approach in which both the overall rotation and the large amplitude bending mode are treated simultaneously. It accounts for the spin-rotation and spin-spin fine couplings, assumed to depend on the large amplitude bending coordinate, as well as for the hyperfine coupling. The new treatment is tested analyzing the available high-resolution data. 336 transitions, involving the ground and first excited vibrational states of the bending mode, are reproduced with a unitless standard deviation of 1.3, using 42 molecular constants. Compared to a previous analysis [Brünken et al., J. Chem. Phys. 123 (2005) 164315], the present analysis is more satisfactory as it accounts for a larger data set and the number of data to number of varied constants ratio is larger. The present theoretical treatment also allows us to retrieve the bending potential and the main kinetic energy term.
\end{abstract}

\section{INTRODUCTION}

Because the methylene radical is a very reactive species difficult to observe, ${ }^{1}$ its first laboratory detection was carried out less than 65 years ago when Herzberg and Shoosmith $^{2}$ first located its spectroscopic signature in a UV spectrum. It was found that in its triplet ground electronic state the radical is bent, unlike initially anticipated, and that its bending angle ${ }^{3}$ is close to $136^{\circ}$. This non-linearity was later confirmed theoretically by Shavitt ${ }^{4}$ in what also was an early success of the then new $a b$ initio quantum chemistry. The methylene radical was further spectroscopically studied as a consequence of its astrophysical relevance. However, due to the fact that the radical has to be produced in situ and that its rotational transitions are in the experimentally difficult terahertz (THZ) and far-infrared (FIR) domains, the spectroscopy of the methylene radical is experimentally very challenging and investigations of its the high-resolution spectrum are not so numerous. High-resolution data have been recorded in the microwave $\mathrm{e}^{5}(\mathrm{MW})$, submillimeter wave $^{6,7}$ (SBMW), THZ, ${ }^{8,9} \mathrm{FIR}^{10}{ }^{10}$ and infrared ${ }^{11-14}$ (IR) domains.

The methylene radical is also very difficult from the theoretical point of view. Due to the very low barrier to linearity ${ }^{15}$ of $1940 \mathrm{~cm}^{-1}$, it is a quasi-linear molecule displaying a very pronounced anomalous centrifugal distortion. ${ }^{16}$ This was confirmed by the spectroscopic measurements which revealed that the Watsontype Hamiltonian ${ }^{17}$ fails to accurately reproduce the methyl radical spectrum. A very large number of highorder distortion terms are needed to account for a small data set. In the case of the ground vibrational state, Sears et al. ${ }^{10}$ were led to use sextic distortion terms for a data set characterized by maximum $N$ and $K_{a}$ values of 5 and 2, respectively. This inadequacy of the Watsontype Hamiltonian is consistent with the very small convergence radius of its polynomial-type expansion with

* laurent.coudert@u-psud.fr respect to the rotational quantum number $K_{a}$. This convergence radius, smaller than in the case of water, ${ }^{18}$ was estimated $^{7}$ to be only 4 .

Alternative theoretical approaches were developed to compute the energy levels of the $\mathrm{CH}_{2}$ radical. These include variational approaches based on the non-rigid bender ${ }^{19,20}$ and the Morse oscillator-rigid bender internal dynamics ${ }^{21}$ Hamiltonians, aided by ab initio calculations and data fitting, ${ }^{15,22-24}$ and effective approaches ${ }^{7}$ where the rotational Hamiltonian of the molecule is approximated by an Euler series. ${ }^{25}$ The variational approaches ${ }^{15,22-24}$ were key to assign the spectrum and to determine the singlet-triplet splittings. They made it possible to reproduce line positions with a $0.02 \mathrm{~cm}^{-1}$ accuracy, even for transitions involving different vibrational states, but these approaches did not achieve experimental uncertainty and did not account for the fine and hyperfine splittings. The effective model of Brünken et al. ${ }^{7}$ accurately computes these splittings and reproduces line positions within experimental uncertainty, but only for transitions belonging to the ground vibrational state.

Non-rigid molecules can be treated efficiently making use of an adiabatic separation between 'fast' small amplitude modes and 'slow' large amplitude modes. ${ }^{19,26}$ This Born-Oppenheimer-type approximation leads to an effective Hamiltonian depending only on the large amplitude coordinates. This approximation was applied to many types of large amplitude motions, including, but not limited to, the large amplitude torsional motion of methanol ${ }^{27,28}$ and hydrogen peroxide, ${ }^{29}$ the large amplitude bending mode of the water molecule, ${ }^{20}$ and the inversion of ammonia. ${ }^{30}$ In this investigation, the fourdimensional Bending-Rotation approach, ${ }^{31-37}$ which relies on the same separation ${ }^{19,26}$ to treat the large amplitude bending mode of the water molecule, is modified to model the rovibrational energies of the $\mathrm{CH}_{2}$ radical. This new approach is applied to the fitting of its spectroscopic data. In addition to the data set considered by Brünken et al. ${ }^{7}$ the fitted data include $148 \nu_{2}$ band transitions reported by McKellar et al. ${ }^{12}$ and by Marshall and McKellar, ${ }^{14} 44$ rotational transitions with field- 
free line position deduced from the laser magnetic resonance (LMR) measurements of Sears et al., ${ }^{10}$ and $10 \nu_{2}$ band transitions with fine structure splittings removed reported by Sears et al. ${ }^{11}$ A total of 336 line positions were reproduced with a unitless standard deviation of 1.3 using 42 molecular constants.

The paper has three remaining sections. In Section II the modified version of the Bending-Rotation approach is introduced. Section III deals with the line position analysis and Section IV is the conclusion.

\section{THEORY}

\section{A. Exact Hamiltonian}

As in the previous version of the Bending-Rotation approach, ${ }^{31-37}$ we start from the exact Hamiltonian of an $\mathrm{XY}_{2}$ triatomic molecule derived considering the overall rotation and the bending mode only. The reference configuration adopted is parameterized by the Radau ${ }^{38,39}$ bending angle $\gamma$ and the two Radau stretching coordinates $R_{1}$ and $R_{2}$. Assuming the latter are constant leads to the fairly simple rovibrational Hamiltonian given in Eqs. (1)-(3) of Coudert. ${ }^{31}$ In the present approach, this approximation is no longer made and the two stretching coordinates $R_{1}$ and $R_{2}$ are assumed $\gamma$-dependent and equal. Attaching the $x y z$ molecule fixed axis system to the molecule so that the $x z$ plane contains the three atoms, the $x$-axis bisecting the angle $\gamma$ and pointing towards the $\mathrm{X}$ atom, the following exact bending-rotation Hamiltonian $H_{\mathrm{b}-\mathrm{r}}$ arises:

$$
H_{\mathrm{b}-\mathrm{r}}=H_{\mathrm{t}}+V(t)+W(t)
$$

where $t=\cos \gamma$ is the large amplitude coordinate for the bending mode; $H_{\mathrm{t}}$ contains kinetic energy terms; $V(t)$ is the potential energy function; and $W(t)$ is the mass dependent contribution to the potential energy function. $H_{\mathrm{t}}$ takes the following expression:

$$
\begin{aligned}
& H_{\mathrm{t}}=P_{t} \frac{B(t)\left(1-t^{2}\right)}{\alpha(t)} P_{t} \\
& \quad+\frac{1}{2} \frac{B(t) N_{x}^{2}}{1-t}+\frac{1}{4} B(t) N_{y}^{2}+\frac{1}{2} \frac{B(t) N_{z}^{2}}{1+t},
\end{aligned}
$$

where $P_{t}$ is the momentum conjugate to $t ; N_{x}, N_{y}$, and $N_{z}$ are molecule fixed components of the rotational angular momentum; $B(t)$ is a $t$-dependent kinetic energy term; and $\alpha(t)$ is:

$$
1+\left(1-t^{2}\right)\left[\frac{B^{\prime}(t)}{B(t)}\right]^{2} .
$$

The volume element to be used for the Hamiltonian in Eq. (1) is $d \Omega d t$, where $d \Omega$ is the usual volume element for the Euler angles. The kinetic energy term $B(t)$ in Eq. (2) can be expressed in terms of the two Radau stretching coordinates as $B(t)=1 /\left(m_{\mathrm{Y}} R^{2}\right)$, where $R=R_{1}=R_{2}$

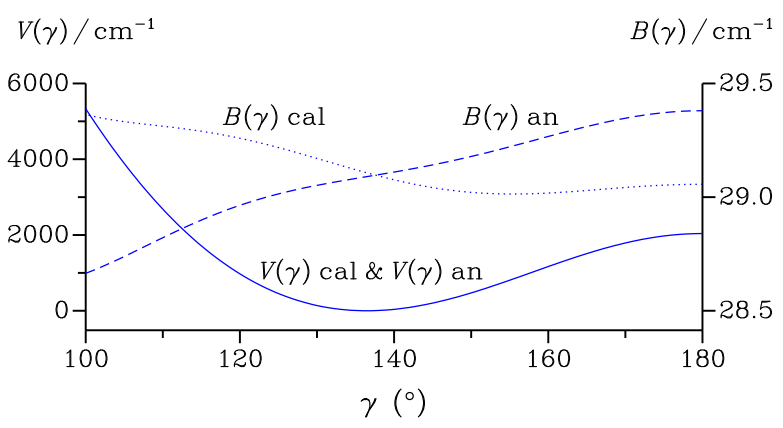

Figure 1. The bending potential $V(\gamma)$ and the kinetic energy term $B(\gamma)$ in $\mathrm{cm}^{-1}$ as functions of the Radau bending angle $\gamma$ in degrees. The left and right scales are to be used for the bending potential and kinetic energy term, respectively. The words 'cal' and 'an' refer to quantities calculated from the potential energy surface of Jensen and Bunker ${ }^{24}$ and retrieved from the line position analysis of Section III, respectively. The differences between the two bending potentials are barely visible in this figure so that only the calculated potential is plotted.

and $m_{\mathrm{Y}}$ is the mass of the $\mathrm{Y}$ atom. The mass dependent contribution to the potential energy function $W(t)$ has a complicated expression. It is given below in the $\alpha^{\prime}(t)=0$ limiting case:

$$
W(t)=\left(1-t^{2}\right)\left[\frac{B^{\prime}(t)^{2}}{B(t)}-B^{\prime \prime}(t)\right]+2 t B^{\prime}(t) .
$$

When the two Radau stretching coordinates are held constant, it can be seen that the exact Hamiltonian of Eq. (1) reduces to that in Eqs. (1)-(3) of Coudert ${ }^{31}$ as $B(t)$ no longer depends on $t, W(t)$ goes to zero, and $\alpha(t)$ goes to unity.

The potential energy function $V(t)$ and the kinetic energy term $B(t)$ were retrieved from the tridimensional potential energy surface of Jensen and Bunker. ${ }^{24}$ The Schrödinger equation for the two stretching coordinates was solved ${ }^{40}$ yielding $t$-dependent stretching energies and eigenfunctions. The latter were used to retrieve the kinetic energy term $B(t)$ taken as the expectation value of $\left(1 / R_{1}^{2}+1 / R_{2}^{2}\right) /\left(2 m_{\mathrm{H}}\right)$. Figure 1 shows the variations of the potential energy function and the kinetic energy term as functions of the Radau bending angle $\gamma$. We can see that the equilibrium value of $\gamma$ is $136.5^{\circ}$, corresponding to a value of $133.4^{\circ}$ for the usual bending angle, and that the barrier to linearity is $2038 \mathrm{~cm}^{-1}$. The total variation of the kinetic energy term $B(\gamma)$ is less than $0.4 \mathrm{~cm}^{-1}$. Its minimum value, $29.01 \mathrm{~cm}^{-1}$, is reached when $\gamma$ is $155.5^{\circ}$. It increases up to $29.06 \mathrm{~cm}^{-1}$ at the linear $\gamma=\pi$ configuration.

The bending-rotation Hamiltonian of Eq. (1) was derived neglecting small amplitude vibrational modes and assuming complete separation ${ }^{19,26}$ between these modes and the large amplitude bending mode. When the separation is not complete, small additional terms arise in the bending-rotation Hamiltonian. A derivation of these terms and of their effects is outside the scope of the 
Table I. Bending-Rotation energies ${ }^{\mathrm{a}}$

\begin{tabular}{rrrrrr}
\hline \hline$v_{2}$ & $N$ & $K_{a}$ & $K_{c}$ & $E^{\mathrm{b}}$ & $E^{\mathrm{c}}$ \\
\hline 0 & 1 & 0 & 1 & 15.64 & 15.68 \\
& 1 & 1 & 1 & 78.32 & 78.43 \\
& 1 & 1 & 0 & 79.51 & 79.61 \\
\hline 1 & 0 & 0 & 0 & 963.1 & 964.58 \\
& 1 & 0 & 1 & 978.6 & 980.16 \\
& 1 & 1 & 1 & 1132.1 & 1134.64 \\
& 1 & 1 & 0 & 1133.4 & 1135.85 \\
\hline \hline
\end{tabular}

a For levels assigned by the bending vibrational quantum number $v_{2}$ and the rotational quantum numbers $N K_{a} K_{c}$, the energies reported by Jensen and Bunker ${ }^{24}$ are compared to those calculated in Section II B without fitting.

b Energy in $\mathrm{cm}^{-1}$ reported by Jensen and Bunker. ${ }^{24}$

c Energy in $\mathrm{cm}^{-1}$ obtained in this work.

present paper and has already been dealt with. ${ }^{41,42}$

\section{B. Bending-rotation energies}

The potential energy function and the kinetic energy term were expanded using a polynomial expansion in $(t-$ $\left.t_{e}\right)^{i}$, where $t_{e}=-0.724966$ is the equilibrium value of $t$ retrieved in the previous section. We have:

$$
V(t)=\sum_{i=2}^{8} V^{i}\left(t-t_{e}\right)^{i},
$$

where $V^{2}, \ldots, V^{8}$ are $21415,-12921,18999,-22259$, $14035,-422$, and $-2290 \mathrm{~cm}^{-1}$, respectively. For the kinetic energy term:

$$
B(t)=\sum_{i=0}^{5} B^{i}\left(t-t_{e}\right)^{i},
$$

where $B^{0}, \ldots, B^{5}$ are $29.107,0.769,0.342,-4.704,6.227$, and $-1.143 \mathrm{~cm}^{-1}$, respectively. The expansions in Eqs. (5) and (6) allow us to reproduce the values calculated in the previous section with root mean square (RMS) deviations of $1.2 \times 10^{-3}$ and $5.7 \times 10^{-5} \mathrm{~cm}^{-1}$, respectively.

The procedure presented in Section 2. $C$ of Coudert ${ }^{32}$ was utilized to obtain bending-rotation energies. The matrix of the bending-rotation Hamiltonian in Eq. (1) is set up using the following bending-rotation basis set functions:

$$
\left|\psi_{v_{2} N k}\right\rangle=\phi_{v_{2}}^{N k}(t)|N k\rangle
$$

where $v_{2}$ is the bending quantum number; $|N k\rangle$ is a usual symmetric top rotational function characterized by the rotational quantum numbers $N$ and $k$ which correspond respectively to the rotational angular momentum and its projection onto the molecule fixed $z$-axis; and $\phi_{v_{2}}^{N k}(t)$ is a bending function. This function is the eigenfunction of the bending Hamiltonian $H_{\mathrm{b}}^{N k}$ arising when diagonal matrix elements of the bending-rotation Hamiltonian between two $|N k\rangle$ rotational functions are taken:

$$
H_{\mathrm{b}}^{N k}=H_{\mathrm{t}}^{N k}+V(t)+W(t),
$$

where:

$$
\begin{aligned}
& H_{\mathrm{t}}^{N k}=P_{t} \frac{B(t)\left(1-t^{2}\right)}{\alpha(t)} P_{t} \\
& \quad+\frac{1}{4} \frac{B(t)\left[N(N+1)-k^{2}\right]}{1-t}+\frac{1}{2} \frac{B(t) k^{2}}{1+t} \\
& \quad+\frac{1}{8} B(t)\left[N(N+1)-k^{2}\right] .
\end{aligned}
$$

The Schrödinger equation for the bending Hamiltonian $H_{\mathrm{b}}^{N k}$ was solved using the bending basis set functions $\theta_{p}^{\alpha, \beta}(t)$ defined in Eq. (4) of Coudert. ${ }^{32}$ A maximum value of $p$ denoted $p_{\max }$ was selected and the values of $\alpha$ and $\beta$ were chosen so that these functions are the eigenfunctions of $H_{\mathrm{t}}^{N k}$ near the $t= \pm 1$ singularities, leading to:

$$
\alpha=\sqrt{\left[N(N+1)-k^{2}\right] / 2} \text { and } \beta=|k| \text {. }
$$

The matrix elements of the bending Hamiltonian $H_{\mathrm{b}}^{N k}$ were evaluated with a Gauss-Jacobi quadrature. Although this is straightforward in the case of potential energy terms, for the $B(t) /(1+t)$ and $P_{t} B(t)\left(1-t^{2}\right) / \alpha(t) P_{t}$ kinetic energy terms, Eqs. (A4)-(A6) should be used because of the $t= \pm 1$ singularities. Diagonalization of the bending Hamiltonian matrix allows us to obtain $\Delta k=0$ matrix elements of the bending-rotation Hamiltonian between two basis set functions of Eq. (7):

$$
\left\langle\psi_{v_{2}^{\prime} N k}\left|H_{\mathrm{b}-\mathrm{r}}\right| \psi_{v_{2} N k}\right\rangle=\delta_{v_{2}^{\prime}, v_{2}} E_{\mathrm{b}}\left(v_{2} N k\right),
$$

where $E_{\mathrm{b}}\left(v_{2} N k\right)$ is the bending energy. $|\Delta k|=2$ matrix elements leads to the following bending matrix elements:

$$
\left\langle\theta_{p^{\prime}}^{\alpha^{\prime}, \beta^{\prime}}|A| \theta_{p}^{\alpha, \beta}\right\rangle
$$

where $A(t)$ is either $B(t) /(1-t)$ or $B(t)$. Such matrix elements can also be obtained from Eqs. (A4)-(A6). The matrix of $H_{\mathrm{b}-\mathrm{r}}$ can now be set up. It is truncated taking a maximum value of the bending quantum number $v_{2}$ denoted $v_{2 \max }$.

Due to the repulsive nature of the bending potential $V(t)$ when the Radau bending angle $\gamma$ decreases, the bending function $\phi_{v_{2}}^{N k}(t)$ becomes vanishingly small when $t \rightarrow+1$. For this reason the value of $\alpha$ for the bending basis set functions is not critical and it was set to a positive value denoted $\alpha_{\max }$, regardless of $N$ and $k$. Using the expansions in Eqs. (5) and (6), taking $\alpha_{\max }=16$, $p_{\max }=23$, and $v_{2 \max }=7$, and ignoring the mass dependent potential $W(t)$, bending-rotation energies were computed and are reported in Table I for $v_{2} \leq 1$ and $N \leq 1$ where they are compared to those of Jensen and Bunker. ${ }^{24}$ The largest discrepancy is $2.6 \mathrm{~cm}^{-1}$ for the $v_{2}=1,1_{11}$ level. 


\section{Fitting Hamiltonian}

A fitting Hamiltonian $H_{\text {fit }}$ is built adding rotational distortion terms ${ }^{32,36}$ to the exact bending-rotation Hamiltonian in Eq. (1) and accounting for the spinrotation and spin-spin couplings. A dependence on the large amplitude coordinate $t$ is assumed for both fine structure interactions along with centrifugal distortion effects. The spin-rotation coupling Hamiltonian with centrifugal distortion terms corresponding to the $A$-reduced form of Brown and Sears ${ }^{43}$ is written:

$$
H_{\mathrm{s}-\mathrm{r}}=\sum_{i=0}^{2}\left(t-t_{e}\right)^{i} H_{\mathrm{s}-\mathrm{r}}^{i},
$$

where:

$$
\begin{aligned}
& H_{\mathrm{s}-\mathrm{r}}^{i}=\frac{1}{2} \sum_{\delta=x y z} \epsilon_{\delta \delta}^{i}\left(N_{\delta} S_{\delta}+S_{\delta} N_{\delta}\right) \\
& \quad+{ }^{S} \Delta_{N}^{i} \mathbf{N}^{2}(\mathbf{N} \cdot \mathbf{S})+\frac{1}{2} S_{\Delta_{N K}^{i}}\left\{\mathbf{N}^{2} N_{z} S_{z}\right. \\
& \left.\quad+N_{z} S_{z} \mathbf{N}^{2}\right\}+{ }^{S} \Delta_{K N}^{i} N_{z}^{2}(\mathbf{N} \cdot \mathbf{S})+{ }^{S} \Delta_{K}^{i} N_{z}^{3} S_{z} \\
& \quad+{ }^{S} \delta_{N}^{i}\left(N_{+}^{2}+N_{-}^{2}\right)(\mathbf{N} \cdot \mathbf{S}) \\
& \quad+\frac{1}{2}{ }^{S} \delta_{K}^{i}\left\{\left(N_{+}^{2}+N_{-}^{2}\right) N_{z} S_{z}\right. \\
& \left.\quad+N_{z} S_{z}\left(N_{+}^{2}+N_{-}^{2}\right)\right\}+\cdots
\end{aligned}
$$

In this equation, $\epsilon_{\delta \delta}^{i}$, with $\delta=x y z$, are molecular constants corresponding to the coefficients of the expansion in terms of $t-t_{e}$ of the diagonal components of the spin-rotation coupling tensor $\boldsymbol{\epsilon}(t) ; S_{x}, S_{y}$, and $S_{z}$ are molecule fixed components of the electron spin operator; and ${ }^{S} \Delta_{N}^{i}, \ldots,{ }^{S} \delta_{K}^{i}$ are the distortion constants of Brown and Sears. ${ }^{43}$ The spin-spin coupling Hamiltonian with centrifugal distortion terms identical to those in Eq. (12) of Brünken et al. ${ }^{7}$ is expressed as:

$$
H_{\mathrm{s}-\mathrm{s}}=\sum_{i=0}^{2}\left(t-t_{e}\right)^{i} H_{\mathrm{s}-\mathrm{s}}^{i},
$$

where:

$$
\begin{aligned}
& H_{\mathrm{S}-\mathrm{s}}^{i}=\frac{1}{2}\left[\alpha^{i}+\alpha_{K}^{i} N_{z}^{2}+\alpha_{N}^{i} \mathbf{N}^{2}, 3 S_{z}^{2}-\mathbf{S}^{2}\right]_{+} \\
& \quad+\frac{1}{2}\left[\beta^{i}+\beta_{K}^{i} N_{z}^{2}+\beta_{N}^{i} \mathbf{N}^{2}, S_{x}^{2}-S_{y}^{2}\right]_{+} .
\end{aligned}
$$

In this equation, $[,]_{+}$is the anticommutator and $\alpha^{i}, \ldots, \beta_{N}^{i}$ are molecular constants. The fitting Hamiltonian can now be written:

$$
\begin{aligned}
& H_{\mathrm{fit}}=H_{\mathrm{t}}+P_{t}\left(1-t^{2}\right) H_{F} P_{t}+\sum_{i=0}^{8}\left(t-t_{e}\right)^{i} H_{i} \\
& +\frac{1}{2} \frac{H_{-}}{1-t}+\frac{1}{2} \frac{H_{+}}{1+t}+H_{\mathrm{s}-\mathrm{r}}+H_{\mathrm{s}-\mathrm{s}}
\end{aligned}
$$

where $H_{i}$, with $i=F,+,-, 0,1, \ldots, 8$ are 12 rotational operators written using the $A$-set distortion terms of the
Watson-type Hamiltonian: ${ }^{17}$

$$
\begin{aligned}
H_{i} & =V^{i}+B_{K}^{i} N_{z}^{2}+B_{N}^{i} \mathbf{N}^{2}+2 b^{i} N_{x y}^{2}-\Delta_{K}^{i} N_{z}^{4} \\
& -\Delta_{K N}^{i} \mathbf{N}^{2} N_{z}^{2}-\Delta_{N}^{i} \mathbf{N}^{4} \\
& -\left[\delta_{K}^{i} N_{z}^{2}+\delta_{N}^{i} \mathbf{N}^{2}, N_{x y}^{2}\right]_{+}+\cdots
\end{aligned}
$$

where $N_{x y}^{2}=N_{x}^{2}-N_{y}^{2}$ and $V^{i}, B_{K}^{i}, \ldots, \delta_{N}^{i}$ are molecular constants including the distortion constants of the Watson-type Hamiltonian $\Delta_{K}^{i}, \ldots, \delta_{N}^{i}$. For $i=+,-$, and 0 , the $V^{i}$ constant is not relevant.

\section{Bending-rotation energies with fine effects}

As in Section II B, we first choose the basis set functions used to set up the matrix of the fitting Hamiltonian in Eq. (17). The bending-rotation basis set functions of Eq. (7) should be replaced by the following bendingrotation-spin basis set functions:

$$
\left|\psi_{v_{2} N k S J}\right\rangle=\phi_{v_{2}}^{N k S J}(t)|N k S J\rangle,
$$

where the rotation-spin function $|N k S J\rangle$ is characterized by the rotational quantum numbers $N$ and $k$, defined as for Eq. (7), by $S$ corresponding to the electron spin, and by $J$ corresponding to the total rotational plus spin angular momentum $\mathbf{J}=\mathbf{N}+\mathbf{S}$; and $\phi_{v_{2}}^{N k S J}(t)$ is a bending wavefunction. This function is the eigenfunction of the bending Hamiltonian $H_{\mathrm{b}}^{N k S J}$ arising when diagonal matrix elements of the fitting Hamiltonian between two $|N k S J\rangle$ rotation-spin functions are taken:

$$
\begin{aligned}
& H_{\mathrm{b}}^{N k S J}=H_{\mathrm{t}}^{N k}+M_{F} P_{t}\left(1-t^{2}\right) P_{t} \\
& \quad+\frac{1}{2} \frac{M_{-}}{1-t}+\frac{1}{2} \frac{M_{+}}{1+t}+\sum_{i=0}^{8}\left(t-t_{e}\right)^{i} \cdot M_{i} \\
& \quad+\sum_{i=0}^{2}\left(t-t_{e}\right)^{i} \cdot\left[M_{\mathrm{s}-\mathrm{r}}^{i}+M_{\mathrm{s}-\mathrm{s}}^{i}\right]
\end{aligned}
$$

where:

$$
\begin{aligned}
& M_{\mathrm{s}-\mathrm{r}}^{i}=\left\langle N k S J\left|H_{\mathrm{s}-\mathrm{r}}^{i}\right| N k S J\right\rangle, \\
& M_{\mathrm{s}-\mathrm{s}}^{i}=\left\langle N k S J\left|H_{\mathrm{s}-\mathrm{s}}^{i}\right| N k S J\right\rangle, \\
& M_{i}=\left\langle N k\left|H_{i}\right| N k\right\rangle,
\end{aligned}
$$

with $i=F,+,-, 0,1, \ldots, 8$. The spin-rotation, spinspin, and rotational matrix elements in Eqs. (21) should be evaluated using the results of Brown and Sears, ${ }^{43}$ Raynes, ${ }^{44}$ and Watson. ${ }^{17}$ The Schrödinger equation for the bending Hamiltonian in Eq. (20) can be solved in the same manner as in Section II B. The bending basis set functions $\theta_{p}^{\alpha, \beta}(t)$ are also used, but the values of $\alpha$ and $\beta$ should be changed:

$$
\begin{aligned}
& \alpha=\sqrt{\frac{B(t=+1)\left[N(N+1)-k^{2}\right] / 2+M_{-}}{B(t=+1)+M_{F}}}, \\
& \beta=\sqrt{\frac{B(t=-1) k^{2}+M_{+}}{B(t=-1)+M_{F}}} .
\end{aligned}
$$


Using the same procedure as in Section II B, the matrix of the bending Hamiltonian of Eq. (20) can be set up and diagonalized allowing us to express the $\Delta N=\Delta k=0$ matrix elements of the fitting Hamiltonian between two basis set functions of Eq. (19) as:

$$
\left\langle\psi_{v_{2}^{\prime} N k S J}\left|H_{\mathrm{fit}}\right| \psi_{v_{2} N k S J}\right\rangle=\delta_{v_{2}^{\prime}, v_{2}} E_{\mathrm{b}}\left(v_{2} N k S J\right),
$$

where $E_{\mathrm{b}}\left(v_{2} N k S J\right)$ is the bending energy. $|\Delta k|=2$ or $|\Delta N|>0$ matrix elements should be evaluated as in Section IIB. A bending matrix element as that in Eq. (12) arises and in addition to the bending operators listed after this equation, operators such as $1 /(1 \pm t)$ and $\left(t-t_{e}\right)^{i}$ should be dealt with. Again, the matrix of the fitting Hamiltonian can be set up and truncated selecting a maximum value of the bending quantum number. The bending-rotation energy levels with fine structure effects which arise after diagonalization are labeled with the bending vibrational quantum number $v_{2}$, the usual rotational quantum numbers $N K_{a} K_{c}$, and $J$ corresponding to the rotational plus spin angular momentum.

\section{E. Hyperfine effects}

The hyperfine coupling Hamiltonian with the same centrifugal distortion terms as in Eq. (11) of Brünken et $a l .{ }^{7}$ takes the form:

$$
\begin{aligned}
H_{\mathrm{hfs}} & =\frac{1}{2}\left[a_{F}+a_{F K} N_{z}^{2}+a_{F N} \mathbf{N}^{2}, \mathbf{I}_{\mathrm{H}} \cdot \mathbf{S}\right]_{+} \\
& +\mathbf{S} \cdot \mathbf{T} \cdot \mathbf{I}_{\mathrm{H}},
\end{aligned}
$$

where $\mathbf{I}_{\mathrm{H}}$ is the nuclear spin and $\mathbf{T}$ is the traceless rank 2 tensor representing the nuclear spin-electron spin dipolar interaction. The Hamiltonian in Eq. (24) depends on 5 molecular constants: $a_{F}, a_{F K}, a_{F N}, T_{z z}$, and $T_{x x}$. Hyperfine energies were taken into account to zeroth order. The coupling scheme to be used is detailed in Section IIIB of Brünken et al. ${ }^{7}$ and matrix elements were evaluated using the results of Bowater et al. ${ }^{45}$

\section{LINE POSITION ANALYSIS}

In the line position analysis, bending-rotation-spin energies were calculated using the results in Section IID. $\alpha_{\max }, p_{\max }$, and $v_{2 \max }$ were set to the same values as in Section II B. The molecular constants involved in the fitting Hamiltonian of Eq. (17) and in the expansion of the kinetic energy term $B(t)$ in Eq. (6) were either varied or constrained to the values calculated in Section II B.

The data set consists of $\mathrm{MW}^{5}{ }^{\mathrm{SBMW}}{ }^{7} \mathrm{THZ}^{8,9}$ and $\mathrm{IR}^{12-14}$ transitions along with ground state combination differences (GSCD) deduced from $\nu_{2}$ band measurements. ${ }^{12,14}$ Also considered were data retrieved from measurements carried out in the FIR and IR domains by Sears et al. ${ }^{10,11}$ with the help of the LMR technique. Field-free wavenumbers, not reported in the investigation of Sears et al., ${ }^{10}$ were deduced from the energy levels given in their Table VIII for the 44 field-free transitions listed in their Table II. In the subsequent investigation of Sears et al., ${ }^{11}$ the authors only report wavenumbers for $\nu_{2}$ band transitions with fine structure splittings removed. The data subsets are listed in Table II and correspond to 336 transitions.

Line positions were introduced in a weighted leastsquares fitting program in which each transition was given a weight equal to the inverse of its experimental uncertainty squared. Unresolved $K$-type doublets in the data reported by Sears ${ }^{13}$ and by Marshall and McKellar ${ }^{14}$ were treated as a single transition. The $2_{11} \leftarrow 2_{02}$ terahertz transition initially reported by Brünken et al. ${ }^{9}$ consists of more than 20 fine and hyperfine structure components. In Brünken et al. ${ }^{7}$ the frequency of these components differ slightly from those initially measured except for the $2_{11}, J, F=3,2 \leftarrow 2_{02}, 3,3$ transition for which there is a $1.2 \mathrm{MHz}$ difference. In the analysis, the newest frequency value ${ }^{7}$ was used for this component while for the remaining ones the frequencies initially measured ${ }^{9}$ were utilized. The SBMW transition measured at $444913.831 \mathrm{MHz}$ by Brünken et al. ${ }^{7}$ was excluded from the analysis because it displayed a too large residual compared to its experimental uncertainty of $30 \mathrm{kHz}$. In preliminary analyses, its residual was $-0.7 \mathrm{MHz}$ leading to a too large value of 3.0 for the unitless standard deviation of the fit. After excluding this transition, the unitless standard deviation decreased by a factor larger than 2. Since the four other fine structure components of this transition are correctly accounted for, it is believed that the frequency reported by Brünken et $a l .{ }^{7}$ is erroneous. The final value of the unitless standard deviation is 1.3 , varying 42 molecular constants.

For each data subset, Table II lists the values of the weighted root mean square (WRMS) and RMS for the observed minus calculated residuals. In the case of a satisfactory analysis, the WRMS and RMS should be respectively close to 1 and to the experimental uncertainty. For the MW, SBMW, and THZ transitions, corresponding to Data Subsets 1 and 2, Table II shows that the least satisfactory results were obtained. The WRMS values are larger than 1 and larger than the values obtained by Brünken et al. ${ }^{7}$ The largest residuals for these data subsets are -1.8 and $-1.2 \mathrm{MHz}$ for respectively the $J, F=1,2 \leftarrow 2,3$ and $1,1 \leftarrow 2,2$ fine and hyperfine components of the $1_{11} \leftarrow 2_{02}$ transition. For the remaining data subsets, the analysis results are satisfactory since the RMS values are on the order or smaller than the experimental uncertainties. For Data Subset 7, corresponding to $\nu_{2}$ band transitions with fine structure splittings removed, the RMS value is much smaller than that obtained by Sears et al. ${ }^{11}$ using the semi-rigid bender model. ${ }^{20}$ For Data Subset 3, the RMS values is also much smaller than that previously reported by Jensen and Bunker. ${ }^{24}$ It should be pointed out, however, that the transitions fitted are not the same since fine structure splittings were ignored by these authors. For Data Subset 6 , the RMS value is smaller than the experimen- 
Table II. Data description and analysis results ${ }^{\mathrm{a}}$

\begin{tabular}{|c|c|c|c|c|c|c|c|}
\hline \multirow[b]{2}{*}{$\#$} & \multirow[b]{2}{*}{ Spectrum } & \multirow[b]{2}{*}{ Data type $^{\mathrm{c}}$} & \multirow[b]{2}{*}{$N$} & \multirow[b]{2}{*}{ Uncert. $^{\mathrm{d}}$} & \multicolumn{3}{|c|}{ WRMS/RMS $^{\mathrm{b}}$} \\
\hline & & & & & This work & Prev. Inv. ${ }^{\mathrm{e}}$ & Ref. \\
\hline 1 & $\mathrm{SBMW}^{7} \mathrm{THZ}^{9}$ & Rot & 14 & $0.020-0.30$ & 2.4 & 0.6 & 7 \\
\hline 2 & $\mathrm{MW}^{5}{ }^{\mathrm{THHZ}}{ }^{8,9}$ & Rot, HFS & 41 & $0.037-0.50$ & 2.2 & 1.3 & 7 \\
\hline 3 & FIR $\mathrm{LMR}^{10}$ & Rot & 44 & 2.0 & 1.0 & 27 & 24 \\
\hline 4 & $\mathrm{IR}^{13}$ & Rot & 17 & 1.0 & 1.9 & 1.4 & 7 \\
\hline 5 & $\mathrm{IR}^{12}{ }^{12} \mathrm{LMR}^{14}$ & GSCD & 62 & 3.0 & 2.7 & 2.6 & 7 \\
\hline 6 & $\mathrm{IR},{ }^{12} \mathrm{IR} \mathrm{LMR}^{14}$ & $\nu_{2}$ & 148 & 2.0 & 1.7 & 1.2 & 14 \\
\hline 7 & IR $\mathrm{LMR}^{11}$ & $\nu_{2}$, No FS & 10 & 1.0 & 0.6 & 119 & 11 \\
\hline
\end{tabular}

${ }^{a}$ For each data subset identified by a number the spectrum recorded, the data type, the number of transitions $N$, the uncertainty used in the analysis, and WRMS/RMS values are given.

b The unitless WRMS is given for Data Subsets $1 \& 2$ and the RMS in $10^{-3} \mathrm{~cm}^{-1}$ for the remaining subsets.

c Rot and $\nu_{2}$ indicate respectively ground state rotational and $\nu_{2}$ band rovibrational transitions. GSCD stands for ground state combination differences. HFS means that hyperfine splittings are observed. No FS means that fine structure splittings are removed.

d The uncertainty used in the analysis in $10^{-3} \mathrm{~cm}^{-1}$ except for Data Subsets 1 \& 2 where it is a range in MHz.

${ }^{\mathrm{e}}$ From the previous investigation cited in the column headed Ref.

tal uncertainty but slightly larger than that obtained by Marshall and McKellar. ${ }^{14}$ In their analysis a Watsontype Hamiltonian and the usual fine structure coupling Hamiltonians were used. The number of varied molecular constants was 50 and exceeds that in the present investigation.

Table III lists fitted values and uncertainties for the 42 varied molecular constants. 9 constants, constrained to values calculated in Section II B, are also listed. Concerning the fine structure coupling constants, non-zero values for $\alpha^{1}$ and $\beta^{1}$ mean that we were able to obtain the dependence of the spin-spin coupling on the large amplitude coordinate. A non-zero value for bending constant $V^{1}$, initially not considered in the bending potential expansion of Eq. (5), suggests that the value of $t_{e}$ calculated in Section II B lacks accuracy. Only $B^{0}$ and $B^{1}$, the two lowest order coefficients of the expansion of the kinetic energy term $B(\gamma)$ could be determined. The remaining constants, $B^{2}$ to $B^{5}$, were constrained to the values calculated in Section II B. The fitted bending potential $V(\gamma)$ and kinetic energy term $B(\gamma)$ are plotted as functions of the Radau bending angle $\gamma$ in Fig. 1, where they are compared with the variations calculated in Section IIB. There is almost no change for the bending potential, but large differences arise for the kinetic energy term. When $\gamma$ increases, the kinetic energy term calculated in Section II B decreases by $0.2 \mathrm{~cm}^{-1}$ while that obtained from the line position increases by $0.7 \mathrm{~cm}^{-1}$. A first explanation of these differences might be a slightly incorrect dependence of the potential energy surface of Jensen and Bunker $^{24}$ on the Radau bending angle $\gamma$. A second explanation might be a failure of the separation between the bending and stretching modes assumed to derive the bending-rotation Hamilton in Section II A. For molecular constants corresponding to the spin-rotation, spinspin, and hyperfine splittings, Table IV shows a comparison between the values obtained in this work and in Brünken et al. ${ }^{7}$ Only spin-rotation and spin-spin coupling constants of Eqs. (13) and (15) corresponding to the low- est order $i=0$ term can thus be compared. We can see that, although the approaches used are quite different, some molecular constants agree within a few \%. Two observed minus calculated tables are available as supplementary material. The first one is for the FIR and IR data; the second one, for the remaining MW, SBMW, and THZ transitions.

\section{CONCLUSION}

It was shown a long time ago that the rovibrational energy levels of quasi-linear molecules can be calculated accurately making use of a simultaneous treatment of the large amplitude mode and of the overall rotation. These ideas were applied in the sixties to the quasi-linear $\mathrm{PH}_{2}$, HCN, HNCO, and HNCS molecules. ${ }^{16,46,47}$ The same ideas were applied later to the methylene radical, ${ }^{11}$ using the semi-rigid bender model, ${ }^{20}$ and to the water molecule using the so-called Bending-Rotation approach. ${ }^{31-37}$

In the present investigation, a new theoretical treatment is applied to the fitting of high-resolution data pertaining to the $\mathrm{CH}_{2}$ radical. This new treatment is an improved version of the Bending-Rotation approach where a more sophisticated parameterization of the reference configuration is utilized. The corresponding exact Hamiltonian, derived in Section II A, displays a more complicated kinetic energy part involving a kinetic energy term denoted $B(t)$ depending on this parameterization. The calculation of the bending-rotation energies, detailed in Section II B, needs to be carried out numerically as matrix elements of the Hamiltonian kinetic energy term no longer have a closed-form expression. A fitting Hamiltonian is built adding rotational distortion terms to this exact Hamiltonian as well as the fine structure spin-rotation and spin-spin couplings which are expanded in terms of the bending coordinate, Section II C. When applied to the fitting of experimental spectroscopic data, this fitting Hamiltonian does not yield rotational constants as 
Table III. Molecular constants ${ }^{\mathrm{a}}$ obtained in the line position analysis

\begin{tabular}{|c|c|c|c|c|c|c|c|c|}
\hline \multicolumn{2}{|c|}{ Constant } & Value & \multicolumn{2}{|c|}{ Constant } & Value & \multicolumn{2}{|c|}{ Constant } & \multirow{2}{*}{$\begin{array}{r}\text { Value } \\
-4.715(42)\end{array}$} \\
\hline$\overline{b^{0}}$ & $\times 10^{3}$ & $8.18(39)$ & $\epsilon_{y y}^{0}$ & $\times 10^{3}$ & $-4.1372(29)$ & $T_{x x}$ & $\times 10^{4}$ & \\
\hline$\Delta_{K}^{0}$ & $\times 10^{2}$ & $3.80(12)$ & ${ }^{9} \Delta_{N}^{0}$ & $\times 10^{7}$ & $3.9(12)$ & & & \\
\hline$\Delta_{K N}^{0}$ & $\times 10^{3}$ & $7.26(50)$ & $S_{\Delta_{N K}^{0}}^{0}$ & $\times 10^{3}$ & $-2.056(83)$ & $V^{1}$ & & $-8.3(13)$ \\
\hline$\Delta_{N}^{0}$ & $\times 10^{4}$ & $-2.028(21)$ & $s_{\Delta_{K N}^{0}}^{0}$ & $\times 10^{3}$ & $2.064(84)$ & $V^{2}$ & $/ 10^{4}$ & $2.1007(22)$ \\
\hline$\delta_{K}^{0}$ & $\times 10^{3}$ & $-1.34(20)$ & $\overrightarrow{S_{\delta_{N}}^{0}}$ & $\times 10^{7}$ & $9.80(96)$ & $V^{3}$ & $/ 10^{4}$ & $-1.3549(8)$ \\
\hline$\delta_{N}^{0}$ & $\times 10^{5}$ & $-2.042(93)$ & & & & $V^{4}$ & $/ 10^{4}$ & $2.337(35)$ \\
\hline$b^{+}$ & $\times 10^{3}$ & $-7.42(35)$ & $\alpha^{0}$ & $\times 10$ & $2.5649(24)$ & $V^{5}$ & $/ 10^{4}$ & $-2.22593^{\mathrm{b}}$ \\
\hline$\Delta_{K N}^{+}$ & $\times 10^{3}$ & $-5.75(32)$ & $\beta^{0}$ & $\times 10^{2}$ & $4.61(14)$ & $V^{6}$ & $/ 10^{4}$ & $1.40354^{\mathrm{b}}$ \\
\hline$H_{K N}^{+}$ & $\times 10^{5}$ & $-8.14(50)$ & $\alpha_{K}^{0}$ & $\times 10^{4}$ & $-4.20(11)$ & $V^{7}$ & $/ 10^{2}$ & $-4.21583^{\mathrm{b}}$ \\
\hline$\Delta_{K}^{1}$ & $\times 10^{2}$ & $1.6(43)$ & $\beta_{K}^{0}$ & $\times 10^{3}$ & $-4.0(14)$ & $V^{8}$ & $/ 10^{3}$ & $-2.29025^{\mathrm{b}}$ \\
\hline$B_{N}^{2}$ & & $-2.055(32)$ & $\alpha_{N}^{0}$ & $\times 10^{5}$ & $1.55(35)$ & $B^{0}$ & $/ 10$ & $2.90902(3)$ \\
\hline$b^{2}$ & & $1.109(29)$ & $\beta_{N}^{0}$ & $\times 10^{5}$ & $-1.75(13)$ & $B^{1}$ & $\times 10$ & $-4.68(90)$ \\
\hline$\Delta_{K N}^{2}$ & $\times 10^{2}$ & $9.62(45)$ & $\alpha^{1}$ & $\times 10$ & $2.45(19)$ & $B^{2}$ & $\times 10$ & $3.41758^{\mathrm{b}}$ \\
\hline$\Delta_{N}^{2}$ & $\times 10^{4}$ & $7.81(28)$ & $\beta^{1}$ & $\times 10$ & $-1.07(40)$ & $B^{3}$ & & $-4.70448^{\mathrm{b}}$ \\
\hline$\delta_{N}^{2}$ & $\times 10^{4}$ & $2.61(36)$ & & & & $B^{4}$ & & $6.22664^{\mathrm{b}}$ \\
\hline & & & $a_{F}$ & $\times 10^{4}$ & $-7.783(70)$ & $B^{5}$ & & $-1.14279^{\mathrm{b}}$ \\
\hline$\epsilon_{z z}^{0}$ & $\times 10^{4}$ & $6.062(69)$ & $a_{F K}$ & $\times 10^{5}$ & $6.33(15)$ & $t_{e}$ & $\times 10$ & $-7.24966^{\mathrm{b}}$ \\
\hline$\epsilon_{x x}^{0}$ & $\times 10^{3}$ & $-5.1431(18)$ & $T_{z z}$ & $\times 10^{4}$ & $9.794(69)$ & & & \\
\hline
\end{tabular}

${ }^{a}$ Constants are given in $\mathrm{cm}^{-1}$ except for $t_{e}$ which is unitless. For fitted constants, uncertainties are given in parentheses in the same units as the last quoted digit.

b Constrained to the value calculated in Section II B.

Table IV. Molecular constants comparison ${ }^{\mathrm{a}}$

\begin{tabular}{lcc}
\hline \hline Constant & This work & Brünken et al. \\
\hline$\epsilon_{z z}^{0}$ & 18.173 & 21.002 \\
$\epsilon_{x x}^{0}$ & -154.186 & -154.061 \\
$\epsilon_{y y}^{0}$ & -124.031 & -124.362 \\
$\alpha^{0}$ & 7689.348 & 7783.390 \\
$\beta^{0}$ & 1382.011 & 1223.949 \\
$a_{F}$ & -23.334 & -22.094 \\
$T_{z z}$ & 29.362 & 30.482 \\
$T_{x x}$ & -14.137 & -15.150 \\
\hline \hline
\end{tabular}

a Zeroth order fine and hyperfine structure constants, in $\mathrm{MHz}$, obtained in this work are compared to those reported by Brünken et $a l^{7}$

the Watson-type Hamiltonian ${ }^{17}$ utilized in usual spectroscopic investigations of semi-ridid molecules. This fitting Hamiltonian allows us to get the variations of various parameters as functions of the bending coordinate. The most important ones being the bending potential $V(t)$ and the kinetic energy term $B(t)$. The latter leads to $R(t)$ the average value of the stretching coordinate through $R(t)=1 / \sqrt{m_{\mathrm{H}} B(t)}$.

In the line position analysis carried out with the new treatment, 336 transitions involving the ground and first excited vibrational states of the bending mode were reproduced with a unitless standard deviation of 1.3, using 42 molecular constants. An SBMW transitions was excluded from the analysis because it displayed a residual much larger than its experimental uncertainty. These results should be compared to those obtained in the pre- vious analysis of Brünken et al. ${ }^{7}$ where 126 transitions belonging to the ground vibrational state were reproduced with a WRMS of 1.28 using 27 molecular constants. With the present analysis, the number of data to number of constant ratio is 8 and has a much more satisfactory value than in Brünken et al. ${ }^{7}$ where it is 4.7. This is consistent with the present model being more physical as it is based on an exact bending-rotation Hamiltonian.

The variations of the potential energy function $V(t)$ and of the kinetic energy term $B(t)$ obtained in the line position analysis are shown in Fig. 1 where they are compared with those calculated from the tridimensional potential energy surface of Jensen and Bunker. ${ }^{24}$ For the potential energy function there is a very good agreement; for the kinetic energy term, discrepancies on the order of $0.5 \mathrm{~cm}^{-1}$ arise and may originate from a slight inaccuracy of the tridimensional potential energy surface ${ }^{24}$ or from a breakdown of the separation between the bending and stretching modes, assumed to derive the BendingRotation Hamiltonian. A more accurate potential energy surface is needed to understand the nature of these discrepancies. Despite this disagreement, it should be stressed that the non-constant kinetic energy term $B(t)$ introduced in this investigation is the key to the satisfactory analysis achieved in this work. Using a constant kinetic energy term as in the previous version of the Bending-Rotation approach ${ }^{31-37}$ would not have allowed us to properly reproduce the spectrum of the methylene radical.

The usefullness of the present approach will be fully established once it is applied to other molecular systems displaying a large amplitude bending mode. In the case 
of the water molecule, going beyond the maximum $J$ value of 30 reached by Coudert al. ${ }^{37}$ should be attempted. In the case of $\mathrm{NH}_{2}$, it should be verified that the highresolution data available for this molecule can be reproduced without using the very high order distortion constants introduced by Martin-Drummel et al. ${ }^{48}$

\section{Appendix A: Bending matrix elements}

The matrix element of a bending operator $A(t)$ between two basis set functions defined in Eq. (4) of Coudert $^{32}$ is equal to:

$$
\begin{aligned}
& \left\langle\theta_{n}^{\alpha_{1} \beta_{1}}|A| \theta_{m}^{\alpha_{2} \beta_{2}}\right\rangle=\int_{-1}^{+1} d t A(t)(1-t)^{\alpha} \\
& \quad \times(1+t)^{\beta} P_{n}^{\left(\alpha_{1}, \beta_{1}\right)}(t) P_{m}^{\left(\alpha_{2}, \beta_{2}\right)}(t) / \sqrt{h_{n} h_{m}} .
\end{aligned}
$$

where $\alpha=\left(\alpha_{1}+\alpha_{2}\right) / 2$ and $\beta=\left(\beta_{1}+\beta_{2}\right) / 2$. Similarly for a bending operator of the form $P_{t} A(t) P_{t}$, the matrix element takes the form:

$$
\begin{aligned}
& \left\langle\theta_{n}^{\alpha_{1} \beta_{1}}\left|P_{t} A P_{t}\right| \theta_{m}^{\alpha_{2} \beta_{2}}\right\rangle=\int_{-1}^{+1} d t A(t)(1-t)^{\alpha-2} \\
& \quad \times(1+t)^{\beta-2} D_{n}^{\left(\alpha_{1}, \beta_{1}\right)}(t) D_{m}^{\left(\alpha_{2}, \beta_{2}\right)}(t) / \sqrt{h_{n} h_{m}},
\end{aligned}
$$

where $\alpha$ and $\beta$ are the same as for Eq. (A1). In agreement with Partridge and Schwenke, ${ }^{49} D_{n}^{(\alpha, \beta)}(t)$ is a polynomial expressed in terms of the Jacobi polynomial $P_{n}^{(\alpha, \beta)}(t)$ and its derivative $P_{n}^{(\alpha, \beta) \prime}(t)$ as:

$$
\begin{aligned}
& D_{n}^{(\alpha, \beta)}(t)=P_{n}^{(\alpha, \beta)}(t)[(\alpha-\beta) / 2+t(\alpha+\beta) / 2] \\
& \quad-\left(1-t^{2}\right) P_{n}^{(\alpha, \beta) \prime}(t) .
\end{aligned}
$$

Evaluating the matrix elements in Eqs. (A1) and (A2) with a $P$-point Gauss-Jacobi quadrature suited for the weight function $(1-t)^{\alpha-1}(1+t)^{\beta-1}$ leads to:

$$
\left.\begin{array}{l}
\left\langle\theta_{n}^{\alpha_{1} \beta_{1}}|A| \theta_{m}^{\alpha_{2} \beta_{2}}\right\rangle \\
\left\langle\theta_{n}^{\alpha_{1} \beta_{1}}\left|P_{t} A P_{t}\right| \theta_{m}^{\alpha_{2} \beta_{2}}\right\rangle
\end{array}\right\}=\sum_{i=1}^{P} \omega_{i} f\left(t_{i}\right)
$$

where $\omega_{i}$ and $t_{i}$ are respectively the weights and nodes. In the case of Eq. (A1), the function $f(t)$ is:

$$
P_{n}^{\left(\alpha_{1}, \beta_{1}\right)}(t) P_{m}^{\left(\alpha_{2}, \beta_{2}\right)}(t)\left(1-t^{2}\right) A(t) / \sqrt{h_{n} h_{m}} .
$$

In the case of Eq. (A2), the function $f(t)$ is:

$$
D_{n}^{\left(\alpha_{1}, \beta_{1}\right)}(t) D_{m}^{\left(\alpha_{2}, \beta_{2}\right)}(t) \frac{A(t)}{\left(1-t^{2}\right)} / \sqrt{h_{n} h_{m}}
$$

Equation (A5) show that $f(t)$ is a finite smooth function of $t$ event if $A(t)$ varies likes $1 /(1 \pm t)$ near $t=\mp 1$. With Eq. (A6), $f(t)$ also is a finite smooth function of $t$ because all the operators arising in the present investigation vary like $\left(1-t^{2}\right)$ near $t= \pm 1$. In both cases the quadrature should lead to accurate results.

\section{SUPPLEMENTARY MATERIAL}

See supplementary material for the observed minus calculated tables of the FIR and IR transitions and of the MW, SBMW, and THZ transitions.

\section{ACKNOWLEDGMENTS}

The author thanks Prof. S. Brünken for helpful discussion about the measurement of the submillimeter wave transitions used in this work.

\section{DATA AVAILABILITY}

The data that support the findings of this study are available from the corresponding author upon reasonable request.
[1] H. Kojima, H. Toyoda, and H. Sugai, App. Phys. Lett. 55, 1292 (1989).

[2] G. Herzberg and J. Shoosmith, Nature 183, 1801 (1959).

[3] G. Herzberg and J. W. C. Johns, J. Chem. Phys. 54, 2276 (1971).

[4] I. Shavitt, Tetrahedron 41, 1531 (1985).

[5] F. J. Lovas., R. D. Suenram, and K. M. Evenson, Astrophys. J. 267, L131 (1983).

[6] H. Ozeki and S. Saito, Astrophys. J. 451, L97 (1995).

[7] S. Brünken, H. S. P. Müller, F. Lewen, and T. F. Giesen, J. Chem. Phys. 123, 164315 (2005).

[8] E. A. Michael, F. Lewen, G. Winnewisser, H. Ozeki, H. Habara, and E. Herbst, Astrophys. J. 596, 1356 (2003).
[9] S. Brünken, E. A. Michael, F. Lewen, T. Giesen, H. Ozeki, G. Winnewisser, P. Jensen, and E. Herbst, Can. J. Chem. 82, 676 (2004).

[10] T. J. Sears, P. R. Bunker, A. R. W. McKellar, K. M. Evenson, D. A. Jennings, and J. M. Brown, J. Chem. Phys. 77, 5348 (1982).

[11] T. J. Sears, P. R. Bunker, and A. R. W. McKellar, J. Chem. Phys. 77, 5363 (1982).

[12] A. R. W. McKellar, C. Yamada, and E. Hirota, J. Chem. Phys. 79, 1220 (1983).

[13] T. J. Sears, J. Chem. Phys. 85, 3711 (1986).

[14] M. D. Marshall and A. R. W. McKellar, J. Chem. Phys. $\mathbf{8 5}, 3716$ (1986). 
[15] P. R. Bunker and P. Jensen, J. Chem. Phys. 79, 1224 (1983).

[16] G. O. Neely, J. Mol. Spectrosc. 27, 177 (1968).

[17] J. K. G. Watson, "Aspects of quartic and sextic centrifugal effects on rotational energy levels," in Vibrational Spectra and Structure, Vol. 6, edited by J. R. Durig (Elsevier, Amsterdam, 1977) Chap. 1, pp. 1-89.

[18] C. Camy-Peyret and J.-M. Flaud, J. Mol. Spectrosc. 59, 327 (1976).

[19] J. T. Hougen, P. R. Bunker, and J. W. C. Johns, J. Mol. Spectrosc. 34, 136 (1970).

[20] P. R. Bunker and B. M. Landsberg, J. Mol. Spectrosc. 67, 374 (1977).

[21] P. Jensen, J. Mol. Spectrosc. 128, 478 (1988).

[22] P. Jensen, P. R. Bunker, and A. R. Hoy, J. Chem. Phys. 77, 5370 (1982).

[23] P. R. Bunker, P. Jensen, W. P. Kraemer, and R. Beardsworth, J. Chem. Phys. 85, 3724 (1986).

[24] P. Jensen and P. R. Bunker, J. Chem. Phys. 89, 1327 (1988).

[25] H. M. Pickett, J. C. Pearson, and C. Miller, J. Mol. Spectrosc. 233, 174 (2005).

[26] D. Lauvergnat, A. Nauts, Y. Justum, and X. Chapuisat, J. Chem. Phys. 114, 6592 (2001).

[27] R. M. Lees and J. G. Baker, J. Chem. Phys. 48, 5299 (1968).

[28] F. C. D. Lucia, E. Herbst, T. Anderson, and P. Helminger, J. Mol. Spectrosc. 134, 395 (1989).

[29] J.-M. Flaud, C. Camy-Peyret, J. W. C. Johns, and B. Carli, J. Chem. Phys. 91, 1504 (1989).
[30] D. Papoušek, J. M. R. Stone, and V. Špirko, J. Mol. Spectrosc. 48, 17 (1973).

[31] L. H. Coudert, J. Mol. Spectrosc. 154, 427 (1992).

[32] L. H. Coudert, J. Mol. Spectrosc. 165, 406 (1994).

[33] L. H. Coudert, J. Mol. Spectrosc. 181, 246 (1997).

[34] R. Lanquetin, L. H. Coudert, and C. Camy-Peyret, J. Mol. Spectrosc. 195, 54 (1999).

[35] L. H. Coudert, Mol. Phys. 96, 941 (1999).

[36] R. Lanquetin, L. H. Coudert, and C. Camy-Peyret, J. Mol. Spectrosc. 206, 83 (2001).

[37] L. H. Coudert, M.-A. Martin-Drumel, and O. Pirali, J. Mol. Spectrosc. 303, 36 (2014).

[38] R. Radau, Ann. Sci. Ecole Normale Supérieure 5, 311 (1868).

[39] B. R. Johnson and W. P. Reinhardt, J. Chem. Phys. 85, 4538 (1986).

[40] C. Gutlé and L. H. Coudert, J. Mol. Spectrosc. 273, 44 (2012).

[41] S. Blasco and D. Lauvergnat, Chem. Phys. Lett. 373, 344 (2003).

[42] D. Lauvergnat and A. Nauts, Chem. Phys. 305, 105 (2004).

[43] J. M. Brown and T. J. Sears, J. Mol. Spectrosc. 75, 111 (1979).

[44] W. T. Raynes, J. Chem. Phys. 41, 3020 (1964).

[45] I. C. Bowater, J. M. Brown, and A. Carrington, Proc. R. Soc. Lond. A. 333, 265 (1973).

[46] R. N. Dixon, Trans. Faraday Soc. 60, 1363 (1964).

[47] J. W. C. Johns, Can. J. Phys. 45, 2639 (1967).

[48] M. A. Martin-Drumel, O. Pirali, and M. Vervloet, J. Phys. Chem. A 118, 1331 (2014).

[49] H. Partridge and D. W. Schwenke, J. Chem. Phys. 106, 4618 (1997). 